Trauma Berufskrankh 2015 • 17[Suppl 1]:164-168 DOI 10.1007/s10039-013-1996-8

Online publiziert: 15. November 2013

(c) Springer-Verlag Berlin Heidelberg 2013
S. Dressel ${ }^{1} \cdot$ J.S. Jarvers ${ }^{1} \cdot$ C. Josten $^{1} \cdot$ T.R. Blattert ${ }^{2}$

${ }^{1}$ Klinik für Unfall-, Wiederherstellungs- und Plastische Chirurgie, Universitätsklinikum Leipzig

${ }^{2}$ Abteilung für Wirbelsäulenchirurgie und Traumatologie, Orthopädische Fachklinik Schwarzach

\title{
Eignung von Zementen auf Kalziumbasis
}

\author{
Im Vergleich zu \\ Polymethylmethacrylatzementen
}

\section{Hintergrund}

Die Ballonkyphoplastie gilt als minimalinvasives Verfahren der Wahl bei der Therapie osteoporotischer Wirbelkörperfrakturen. Ihre Vorteile bestehen in einer Schmerzreduktion unmittelbar postoperativ [11], einer frühen Mobilisation [11] sowie einem Ausgleich kyphotischer Fehlstellungen [7].

Vor- und Nachteile

der Zementarten

Bisher gilt Zement auf PMMA-Basis (PMMA: Polymethylmethacrylat) als Goldstandard unter den Augmentationsmaterialien [7]. Er weist jedoch einige Nachteile auf: Zum einen kann er, bedingt durch hohe Aushärtungstemperaturen, thermische Schäden benachbarter Organe hervorrufen [5]. Zum anderen sind systemische Nebenwirkungen nicht polymerisierter toxischer Monomere beschrieben [16]. Neben diesen beiden Komplikationen führte seine fehlende ossäre Integrierbarkeit [12] dazu, dass Zemente auf CaP-Basis (Ca-P: Kalziumphosphat) mehr in den Focus rückten. Diese haben den Vorteil, dass sie resorbierbar sind und $\mathrm{zu}$ eigener Knochenstruktur umgebaut werden [6]. Außerdem erfolgt die Aushärtung nur knapp über Körpertemperatur [14]. Die bisher untersuchten Ca-P-Zemente wiesen eine $\mathrm{zu}$ geringe $\mathrm{Zug}$ - und Biegeelastizität auf [2]. Außerdem wurde als Komplikation eine Zementauswaschung beschrieben [2].
Mit Xeraspine $\odot$ entwickelte die Firma DOXA einen Zement auf Kalziumbasis, der mit seinen zusätzlichen Aluminiumbestandteilen mehr Stabilität durch bessere Bindung von Wasser im Kern verspricht und damit eine mögliche Alternative zum Goldstandard darstellt.

\section{Eigenschaften von Xeraspine $\odot$}

\section{Chemische Beschaffenheit und Verarbeitung}

Xeraspine $\odot$ ist ein biointegratives Augmentationsmaterial und besteht aus Kalziumaluminat und Zirkoniumdioxidpulver. Nach Zugabe einer Flüssigkeit auf Wasserbasis kommt es zu einer chemischen Reaktion in Form einer Kristallisation. Die Verarbeitungszeit beträgt $7 \mathrm{~min}$, die Aushärtung in situ erfolgt knapp über Körpertemperatur. PMMA besitzt im Gegensatz dazu eine Verarbeitungszeit von 10 min und härtet aufgrund der exothermen Reaktion, welche in Form einer Polymerisation abläuft, in situ bei $100^{\circ} \mathrm{C}$ aus [10].

Kalziumaluminat bindet wesentlich mehr Wasser als der konventionelle Ca-PZement, was sich in seiner größeren Festigkeit widerspiegelt [10].

\section{Druck- und Biegeelastizität}

In einer an der Universität Uppsala durchgeführten Studie von Persson u. Axén [15] wurden die verschiedenen Zementarten in $37^{\circ} \mathrm{C}$ warmes PBS („phosphate buffered saline") für einen Zeitraum zwischen $24 \mathrm{~h}$ und 3 Monaten eingelegt. Dabei konnte gezeigt werden, dass Xeraspine $\odot$ im Vergleich zu PMMA bezüglich der Druckund Biegefestigkeit annähernd gleiche Ergebnisse erzielte. Im Vergleich zu Ca-P war die mechanische Festigkeit signifikant höher (• Tab. 1).

\section{Biointegrierbarkeit}

Uhlin et al. [17] untersuchten die Biointegrierbarkeit des Xeraspine@-Zements im Vergleich zu PMMA an Schafen. Hierfür applizierten sie die jeweiligen Zemente in Wirbelkörper lebender Tiere und analysierten diese post mortem unter dem Elektronenmikroskop. Dabei konnten sie beobachten, dass sich Ca-Al (Kalziumaluminat) sehr gut in den Knochen integriert hatte und kein Spalt zwischen diesem und dem Zement nachweisbar war. Im Gegensatz dazu fand man bei PMMA zwischen Knochen und Zement einen Abstand von $10 \mu \mathrm{m}$ [17]. Damit war es erstmals gelungen, einen Zement auf Kalziumbasis herzustellen, der die Stärke der guten Biointegrierbarkeit mit den guten physikalischen Eigenschaften von PMMA - hinsichtlich der Druck- und Biegefestigkeit - zumindest in experimentellen Untersuchungen vereint. Ob sich diese experimentell nachgewiesenen guten mechanischen Eigen-

Ergebnisse der vorliegenden Studie wurden bereits publiziert unter Dressel S, Jarvers JSG, Josten C, Blattert TR (2012) Percutaneous balloon kyphoplasty in osteoporotic vertebral body fracture with a calcium aluminate ceramic (xeraspine $\odot$ ). Eur Musculoskelet Rev 7(4):209-212 Diese Studie wurde finanziell von DOXA, Schweden, unterstützt. 
Tab. 1 Ergebnisse des Kalziumaluminatzements vs. PMMA a und Ca-Pa

\begin{tabular}{llll}
\hline & Xeraspine $\odot$ & PMMA & Ca-P \\
\hline Verarbeitungszeit (min) & 7 & 10 & 3 \\
\hline $\begin{array}{l}\text { Aushärtungstemperatur } \\
\left({ }^{\circ} \mathrm{C}\right)\end{array}$ & 56 & 100 & 37 \\
\hline Druckfestigkeit (MPa) & 90 & 80 & 55 \\
\hline Biegeelastizität (MPa) & 55 & 60 & 20 \\
\hline Biokompatibilität & Gut & Schlecht & Gut \\
\hline CA-P Kalziumphosphat, $P M M A$ Polymethylmethacrylat, ${ }^{2}$ Ausgewählte Daten aus Persson u. Axén [15]
\end{tabular}

Tab. 2 Ergebnisse der radiologischen Parameter

\begin{tabular}{|c|c|c|c|c|c|}
\hline \multicolumn{2}{|c|}{$\begin{array}{l}\text { Untersuchungs- } \\
\text { zeitpunkt }\end{array}$} & $\begin{array}{l}\text { Fraktur- } \\
\text { typ }\end{array}$ & $\begin{array}{l}\text { Vordere Wirbelkör- } \\
\text { perhöhe }(\mathrm{mm})\end{array}$ & $\begin{array}{l}\text { Kyphosewinkel } \\
\left({ }^{\circ}\right)\end{array}$ & $\begin{array}{l}\text { Wiederauf- } \\
\text { richtung (\%) }\end{array}$ \\
\hline \multirow{2}{*}{\multicolumn{2}{|c|}{$\begin{array}{l}\text { Präope- } \\
\text { rativ }\end{array}$}} & A1.3 & $\begin{array}{l}23,0 \\
(n=12)\end{array}$ & $\begin{array}{l}-5,92 \\
(n=12)\end{array}$ & \\
\hline & & A3.1 & $\begin{array}{l}23,2 \\
(n=17)\end{array}$ & $\begin{array}{l}-5,41 \\
(n=17)\end{array}$ & \\
\hline \multirow[t]{12}{*}{$\begin{array}{l}\text { Post- } \\
\text { operativ }\end{array}$} & \multirow{2}{*}{$\begin{array}{l}\text { Direkt } \\
\text { post- } \\
\text { operativ }\end{array}$} & A1.3 & $\begin{array}{l}28,8 \\
(n=12, p=0,002)\end{array}$ & $\begin{array}{l}-1,58 \\
(n=12, p=0,003)\end{array}$ & $\begin{array}{l}12,20 \\
(n=17, p=0,006)\end{array}$ \\
\hline & & A3.1 & $\begin{array}{l}27,4 \\
(n=17, p<0,001)\end{array}$ & $\begin{array}{l}0,47 \\
(n=17, p=0,003)\end{array}$ & $\begin{array}{l}8,20 \\
(n=17, p=0,013)\end{array}$ \\
\hline & \multirow[t]{2}{*}{6 Wochen } & A1.3 & $\begin{array}{l}23,1 \\
(n=12, n . s .)\end{array}$ & $\begin{array}{l}-3,58 \\
(n=12, p=0,027\end{array}$ & $\begin{array}{l}11,92 \\
(n=12, p=0,023)\end{array}$ \\
\hline & & A3.1 & $\begin{array}{l}20,2 \\
(n=17, n . s .)\end{array}$ & $\begin{array}{l}-1,59 \\
(n=17, \text { n.s. })\end{array}$ & $\begin{array}{l}-6,80 \\
(n=17, \text { n.s. })\end{array}$ \\
\hline & \multirow[t]{2}{*}{3 Monate } & A1.3 & $\begin{array}{l}25,0 \\
(n=11, \text { n.s. })\end{array}$ & $\begin{array}{l}-2,36 \\
(n=11, \text { n.s. })\end{array}$ & $\begin{array}{l}6,00 \\
(n=11, \text { n.s. })\end{array}$ \\
\hline & & A3.1 & $\begin{array}{l}20,7 \\
(n=13, \text { n.s. })\end{array}$ & $\begin{array}{l}-3,77 \\
(n=13, \text { n.s. })\end{array}$ & $\begin{array}{l}-9,36 \\
(n=16, \text { n.s. })\end{array}$ \\
\hline & \multirow[t]{2}{*}{6 Monate } & A1.3 & $\begin{array}{l}27,3 \\
(n=10, p=0,002)\end{array}$ & $\begin{array}{l}-4,20 \\
(n=10, \text { n.s. })\end{array}$ & $\begin{array}{l}5,82 \\
(n=16, \text { n.s. })\end{array}$ \\
\hline & & A3.1 & $\begin{array}{l}21,8 \\
(n=16, \text { n.s. })\end{array}$ & $\begin{array}{l}-2,43 \\
(n=16, \text { n.s. })\end{array}$ & $\begin{array}{l}-5,84 \\
(n=16, \text { n.s. })\end{array}$ \\
\hline & \multirow[t]{2}{*}{$\begin{array}{l}12 \\
\text { Monate }\end{array}$} & A1.3 & $\begin{array}{l}26,4 \\
(n=10, p=0,01)\end{array}$ & $\begin{array}{l}-5,50 \\
(n=10, \text { n.s. })\end{array}$ & $\begin{array}{l}8,49 \\
(n=10, n . s .)\end{array}$ \\
\hline & & A3.1 & $\begin{array}{l}20,1 \\
(n=13, \text { n.s. })\end{array}$ & $\begin{array}{l}-4,62 \\
(n=13, \text { n.s. })\end{array}$ & $\begin{array}{l}-6,70 \\
(n=13, \text { n.s. })\end{array}$ \\
\hline & \multirow[t]{2}{*}{$\begin{array}{l}24 \\
\text { Monate }\end{array}$} & A1.3 & $\begin{array}{l}23,5 \\
(n=10, n . s .)\end{array}$ & $\begin{array}{l}-7,10 \\
(n=10, \text { n.s. })\end{array}$ & $\begin{array}{l}8,45 \\
(n=10, \text { n.s. })\end{array}$ \\
\hline & & A3.1 & $\begin{array}{l}18,5 \\
(n=12, n . s .)\end{array}$ & $\begin{array}{l}-3,97 \\
(n=12, \text { n.s. })\end{array}$ & $\begin{array}{l}-7,11 \\
(n=12, n . s .)\end{array}$ \\
\hline
\end{tabular}

schaften auch in vivo am Patienten bestätigen und demzufolge die Refrakturrate tatsächlich in der Praxis gesenkt werden konnte, sollte diese Studie zeigen.

\section{Methoden}

Wir führten eine prospektive klinische Studie über einen Zeitraum von 2 Jahren mit 24 Patienten der Klinik für Unfall-, Wiederherstellungs- und Plastische Chirurgie des Universitätsklinikums Leipzig durch, welche 29 Wirbelkörperfrakturen erlitten hatten, die mittels Xeraspine@-Zement kyphoplastiert wurden.

Die Operation erfolgte in Standardkyphoplastietechnik in Bauchlage, Intubationsnarkose und mit transpedikulärem Zugang.

\section{Ein- und Ausschlusskriterien}

$\mathrm{Zu}$ den Einschlusskriterien zählten 1 oder 2 symptomatische Wirbelkörperfrakturen zwischen BWK8 (Brustwirbelkörper 8) und LWK4 (Lendenwirbelkörper 4) mit Indikation zur Kyphoplastie, ein Al- ter zwischen 50 und 90 Jahren, ein T-Score $<-2,5$ sowie ein initialer Wert auf der VAS (visuelle Analogskala) von $>50$.

Ausschlusskriterien waren eine zusätzliche dorsale Instrumentierung, das Vorliegen einer pathologischen Fraktur, Tumorerkrankung, Herzrhythmusstörungen sowie andere relevante Begleiterkrankungen.

\section{Untersuchungsaspekte}

Die Patienten wurden präoperativ, postoperativ, nach 6 Wochen sowie nach 3, 6, 12 und 24 Monaten hinsichtlich folgender Aspekte untersucht: mit Hilfe der VAS und des ODI („Oswestry disability index“) wurden die subjektiv empfundenen Schmerzen erfragt, außerdem wurden alle Patienten zu allen Nachuntersuchungszeitpunkten einer konventionellen Röntgenuntersuchung unterzogen, anhand derer die Wirbelkörperhöhe, der Kyphosewinkel und die prozentuale Wiederaufrichtung bestimmt werden konnten. $\mathrm{Zu}$ sätzlich veranlassten wir präoperativ sowie nach 6, 12 und 24 Monaten einen CTScan (CT: Computertomographie) der Frakturregion.

Die statistische Auswertung erfolgte mit den Programmen Office 2007 EXCEL (Firma Microsoft, USA) und SPSSStatistics 20.0 für OS Mac (Firma IBM). Die Signifikanz wurde mittels EinstichprobenT-Test errechnet, das Signifikanzniveau lag bei $\mathrm{p}<0,05$. Die in den Nachuntersuchungen bestimmten Werte für VAS und ODI sowie die verschiedenen erhobenen radiologischen Parameter wurden im Verlauf auf signifikante Unterschiede in Bezug zu den präoperativ erhobenen Werten getestet. Alle radiologischen Parameter wurden anhand der konventionellen Röntgenaufnahmen bestimmt.

\section{Ergebnisse}

Von den 23 Patienten waren 14 (61\%) weiblichen und 9 (39\%) männlichen Geschlechts. Das Durchschnittsalter betrug 73,55 Jahre (Minimum 61 Jahre, Maximum 84 Jahre).

Insgesamt wurden 29 Wirbelkörper kyphoplastiert, wobei der BWK12 mit $34,5 \%$ am häufigsten betroffen war, gefolgt vom LWK1 mit 24,1\%. 
Von uns wurden 12 Frakturen als A1.3 (41,4\%) und 17 Frakturen als A3.1 (58,6\%) nach Magerl et al. [13] klassifiziert. Bei 19 Patienten augmentierten wir jeweils 1 und bei 5 Patienten jeweils 2 Wirbelkörper. Als Füllmaterial diente Kalziumaluminatzement. Das durchschnittliche Füllungsvolumen betrug 6,3 ml je Wirbelkörper (Minimum: $5 \mathrm{ml}$; Maximum $9 \mathrm{ml}$ ).

Die Krankenhausliegedauer belief sich im Durchschnitt auf 9,6 Tage.

In der Nachuntersuchungszeit verstarben 2 Patienten, 5 Patienten brachen die Studie vorzeitig ab.

\section{Intraoperative Komplikationen}

Bei 7 der 29 versorgten Wirbelkörper kam es zu einem Zementaustritt, wobei es sich 2-mal um A1.3- und 5-mal um A3.1-Frakturen handelte. Dabei trat der Zement 2-mal nach epidural, 2-mal venös und 3-mal nach paravertebral aus. Keiner der Patienten zeigte neurologische Symptome.

\section{Postoperative Komplikationen}

Innerhalb des Nachuntersuchungszeitraums kam es bei 7 Patienten zu einer Refraktur, wovon 2 erneut operativ versorgt werden mussten. Außerdem wurden 2 Frakturen angrenzender Wirbelkörper beobachtet. Ein Patient erlitt im Verlauf eine neue Fraktur eines entfernt liegenden Wirbelkörpers.

Bei einem Fall kam es am 6. postoperativen Tag zur Bildung eines paravertebralen Hämatoms auf Höhe des ehemals frakturierten Wirbelkörpers, sodass eine operative Ausräumung notwendig war (• Abb. 1).

\section{Radiologische Parameter}

Im Mittel betrug die Höhe der Vorder-, Mittel- und Hinterkanten der frakturierten Wirbelkörper präoperativ $26,2 \mathrm{~mm}$; $23,1 \mathrm{~mm}$ und $28,8 \mathrm{~mm}$. Intraoperativ gelang eine Wiederaufrichtung der Vorderkante um 2,6 mm ( $\mathrm{p}=0,006)$, der Wirbelkörpermitte um $4,8 \mathrm{~mm}(\mathrm{p}<0,001)$ und der Hinterkante um 3,3 mm $(\mathrm{p}<0,001)$. Im Vergleich der Frakturklassifikationen konnte jedoch nur bei den A3.1-Frakturen eine signifikante Wiederaufrichtung

Trauma Berufskrankh 2015 - 17[Suppl 1]:164-168 DOI 10.1007/s10039-013-1996-8

(c) Springer-Verlag Berlin Heidelberg 2013

\section{S. Dressel · J.S. Jarvers · C. Josten · T.R. Blattert Eignung von Zementen auf Kalziumbasis. Im Vergleich zu Polymethylmethacrylatzementen}

\section{Zusammenfassung}

Hintergrund. Die Ballonkyphoplastie ist ein minimalinvasives Verfahren bei Wirbelkörperfrakturen. Bisher gilt Polymethylmethacrylat (PMMA) als Goldstandard unter den Augmentationsmaterialien. Seine Schwächen sind die fehlende Biokompatibilität und die schlechte ossäre Integrierbarkeit, weshalb Alternativen im Bereich biokompatibler Zemente gesucht wurden.

Studiendesign. In einem Zeitraum von 2 Jahren wurden 23 Patienten mit 29 Wirbelkörperfrakturen, welche mittels eines neuen Kalziumaluminatzements kyphoplastiert wurden, untersucht. Intraoperativ konnte eine Wiederaufrichtung der Wirbelkörperhöhe von 4,8 mm erreicht werden. Die Kyphose wurde um $4,6^{\circ}$ korrigiert. Es zeigte sich ein signifikanter Rückgang der Schmerzen. Zementspezifische Komplikationen waren: Refrakturen $(n=7)$, neue Frakturen angrenzender Wirbelkörper $(n=2)$ und Zementaustritte $(n=7)$. Zementauswaschung wurde nicht beobachtet.

Schlussfolgerung. Eine routinemäßige Anwendung des Xeraspine $\odot-Z e m e n t s ~ k a n n$ derzeit nicht empfohlen werden. Im Vergleich zu PMMA besitzt er schlechtere biomechanische Eigenschaften, außerdem zeigten sich eine höhere Refrakturierungsrate und ein schlechteres intraoperatives Handling.

\section{Schlüsselwörter}

Kyphoplastie · Biokeramik · Kalziumzement · Biokompatibilität · Augmentationsmaterial

\section{Suitability of calcium phosphate cements. Compared to polymethylmethcrylate cements}

\section{Abstract}

Background. Balloon kyphoplasty is a minimally invasive surgical technique to treat spinal fractures. So far, polymethylmethacrylate (PMMA) is applied as the first choice augmentation material. However, it is characterized by limited biocompatibility and a lack of osseous integration. Therefore, intensive research was performed to identify other cements based on biomaterials.

Study design. In this prospective clinical study, we investigated 23 patients with 29 vertebral body fractures over 2 years who were treated with a new bone cement based on calcium aluminate ceramic (Xeraspine()) by means of balloon kyphoplasty. Intraoperatively, height restoration of the body center of $4.8 \mathrm{~mm}$ and of the kyphosis angle by $4.6^{\circ}$ was achieved. There was a significant pain relief. Complications specifically associated with the cement were refracture of the augmented vertebra $(n=7)$, new fracture in adjacent vertebral bodies $(n=2)$, and extrusion of cement $(n=7)$. Cement washout was not observed.

Conclusion. Routine use of the Xeraspine $\odot$ cement is not currently recommended for kyphoplasty. Compared with PMMA, the biomechanical properties are worse. Moreover there is a higher refracture rate and subjectively worse intraoperative handling.

\section{Keywords}

Kyphoplasty - Bioceramic - Calcium cement . Biocompatible materials · Augmentation material erzielt werden. Bereits 6 Wochen postoperativ war bei Frakturen beider Klassifikationen insgesamt eine deutliche Sinterung eingetreten, sodass keine Signifikanz der Höhen im Vergleich zum präoperativen Befund zu verzeichnen war (• Tab. 2).

Um eine Aussage über die Wiederaufrichtung der frakturierten Wirbelkörper treffen zu können, bestimmten wir den Kyphosewinkel. Direkt postoperativ waren sowohl die betroffenen Wir- belkörper der A1.3-Frakturen als auch die der A3.1-Frakturen signifikant aufgerichtet (A1.3 postoperativ um 4,34 ${ }^{\circ}$, A3.1 um $\left.4,94^{\circ}\right)$. Bereits 6 Wochen nach der Operation war eine deutliche Resinterung $\mathrm{zu}$ verzeichnen, sodass lediglich für Frakturen vom A3.1-Typ noch signifikante Werte zu messen waren ( $\bullet$ Tab. 2).

Ein weiterer Parameter zur Beurteilung der Wiederaufrichtung ist der prozentuale Vergleich von prä- und postope- 


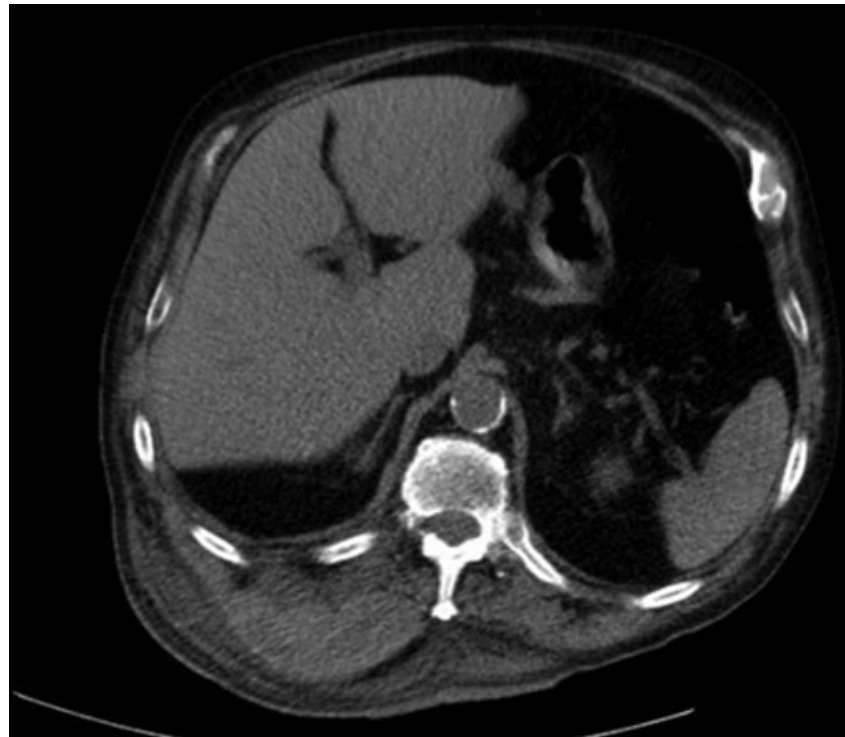

rativen Höhen des frakturierten Wirbelkörpers, jeweils bezogen auf den Nachbarwirbel. Hierbei zeigte sich ebenfalls postoperativ eine signifikante Wiederaufrichtung für beide Frakturtypen (A1.3 $12,2 \%, A 3.18,2 \%$ ), aber auch hier ließ sich 6 Wochen nach dem Eingriff eine Sinterung v. a. für die A3.1-Frakturen nachweisen (- Tab. 2).

\section{Klinische Parameter}

Anhand der Ergebnisse der VAS ergab sich ein Rückgang der Schmerzsymptomatik von im Mittel 77,5 mm präoperativ auf 16,4 mm unmittelbar postoperativ, der über den weiteren Nachuntersuchungszeitraum konstant blieb [nach 24 Monaten 10,6 mm (A1.3) und $20 \mathrm{~mm}$ (A3.1)].

Auch die auf die verschiedenen Alltagsaktivitäten bezogenen Schmerzen, welche mit Hilfe des ODI erfasst wurden, konnten von präoperativ im Mittel 74,3\% auf unmittelbar postoperativ $24,5 \%$ (A1.3) und $26,4 \%$ (A3.1) gesenkt werden. Im Verlauf waren die Werte weiter konstant niedrig mit im Mittel 18,3\% nach 24 Monaten (• Abb. 2).

\section{Diskussion}

Im Rahmen der Kyphoplastie zur Therapie osteoporotischer Wirbelkörperfrakturen wird bevorzugt PMMA verwendet [7]. Durch den Einsatz von biokompatiblen Zementen auf Kalziumbasis wird jedoch ein Material appliziert, das sich den
Abb. $1<$ Paravertebrales Hämatom

\section{Schwächen von Xeraspine $\odot$ gegenüber PMMA}

Die Verarbeitungszeit von Xeraspine $@$ ist etwas kürzer als die von PMMA, weshalb es schneller appliziert werden muss und, bezogen auf das intraoperative Handling, subjektiv schlechter abschneidet als PMMA-Zement. Der Einsatz von Xeraspine $\bigodot$ erfordert, wie die herkömmlichen Zemente auf Ca-P-Basis, vom Operateur eine größere Erfahrung und insgesamt mehr Sicherheit in der Beherrschung des Verfahrens. Dies erschwert das Erlernen der Operationstechnik der Kyphoplastie durch einen unerfahrenen Operateur.

Am meisten Gewicht als Schwäche von Xeraspine $\odot$ aber hat die hohe Komplikationsrate - mit 73,9\% gesamt und 13\% klinisch relevanten Komplikationen (im Vergleich dazu in der Literatur allgemein angegebene Komplikationsraten für eine Kyphoplastie: 16,3\% [19] gesamt, 0,4\% [3] relevant). Von allen beschriebenen Komplikationen fiel die hohe Refrakturierungsrate mit 24\% (im Vergleich: PMMA $<1 \%$ [18]) besonders auf.

Die Ergebnisse für den Kyphosewinkel und die prozentuale Wiederaufrichtung von Xeraspine $\odot$ waren im Vergleich zu PMMA schlechter.

Der Vorteil der Biointegrierbarkeit allein rechtfertigt einen Ersatz von Xeraspine` nicht - zuerst müssten die biomechanischen Eigenschaften von Xeraspine@ weiter verbessert werden.

\section{Kernaussagen} belkörpern keine Zementauswaschungeine sonst typische Komplikation bei CaP-Zement - mehr zu beobachten.

\section{Vorteile von Xeraspine $\odot$ gegenüber PMMA}

Aus den Studienergebnissen lässt sich eine deutliche Überlegenheit von Xeraspine $\subset$ bezogen auf die Ergebnisse der VAS und ODI im Vergleich zu PMMA ableiten [4]. Da allerdings bei allen Zementarten eine signifikante Schmerzreduktion nachgewiesen werden konnte, rechtfertig dies den Einsatz einer speziellen Zementart, bezogen auf den Aspekt Schmerz, nicht.
- Kyphoplastie/Vertebroplastie sind minimalinvasive Verfahren der Wahl in der Behandlung von Wirbelkörperfrakturen.

- PMMA ist bisher Goldstandard, weist jedoch Defizite in Biokompatibilität und Osteointegration auf.

- Ca-P-Zemente sind aufgrund ihrer schlechten biomechanischen Eigenschaften und Wasserlöslichkeit (Zementauswaschung) als kritisch anzusehen.

- Bei Xeraspine@ kommt es nicht zur Zementauswaschung, jedoch ist die Biomechanik weiter unzureichend.

- PMMA bleibt somit weiter Goldstandard. 


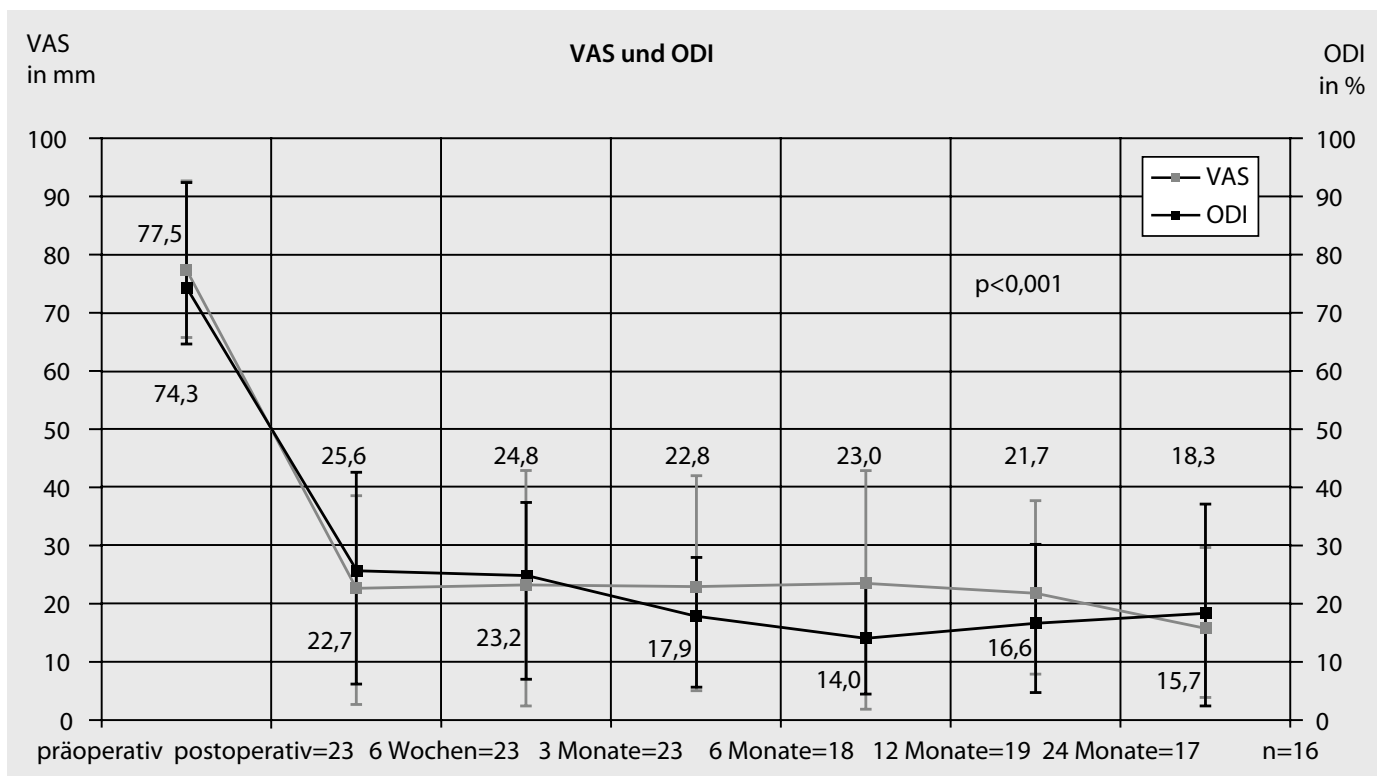

Abb. 24 Ergebnisse von VAS (visuelle Analogskala) und ODI („Oswestry disability index")

\section{Fazit für die Praxis}

- Innovationen in Richtung praxistauglicher biointegrativer Augmentationsmaterialen gibt es.

- Aufgrund weiterhin insuffizienter biomechanischer Eigenschaften auch moderner Zemente auf Kalziumbasis bleibt PMMA Goldstandard für die Zementaugmentation im Rahmen der operativen Versorgung der osteoporotischen Wirbelkörperfraktur.

\section{Korrespondenzadresse}

\section{Prof. Dr. T.R. Blattert}

Abteilung für Wirbelsäulenchirurgie und Traumatologie,

Orthopädische Fachklinik Schwarzach, Dekan-Graf-Straße 2-6, 94374 Schwarzach thomas.blattert@ofks.de

\section{Einhaltung ethischer Richtlinien}

Interessenkonflikt. S. Dressel, J.S. Jarvers, C. Josten und T.R. Blattert geben an, dass kein Interessenkonflikt besteht.

\section{Alle im vorliegenden Manuskript beschriebenen} Untersuchungen am Menschen wurden mit Zustimmung der zuständigen Ethik-Kommission, im Einklang mit nationalem Recht sowie gemäß der Deklaration von Helsinki von 1975 (in der aktuellen, überarbeiteten Fassung) durchgeführt. Von allen beteiligten Patienten liegt eine Einverständniserklärung vor.

The supplement containing this article is not sponsored by industry.

\section{Literatur}

1. Belkoff SM, Mathis JM, Jasper LE et al (2001) An ex vivo biomechanical evaluation of a hydroxyapatite cement for use with vertebroplasty. Spine 26:1542-1546

2. Blattert TR, Weckbach A (2004) Kalziumphosphat vs. Polymethylmethacrylat - Erste Ergebnisse einer prospektiven, randomisierten, klinischen Vergleichsstudie zur perkutanen Ballonkyphoplastie. Trauma Berufskrankh 6:(2)S273-S278

3. Boszczyk BM, Bierschneider M, Hauck S et al (2004) Kyphoplastik im konventionellen und halboffenen Verfahren. Orthopade 33(1):13-21

4. Delank KS, Röllinghoff M, Eysel-Gosepath K et al (2010) Degeneration und Osteoporose der Wirbelsäule. Orthopade 4:425-431

5. Deramond H, Wright NT, Belkoff SM (1999) Temperature elevation caused by bone cement polymerization during vertebroplasty. Bone [Suppl 2] 25:17S-21S

6. Friedman CD, Costantino PD, Takagi S et al (1998) BoneSource hydroxyapatite cement: a novel biomaterial for craniofacial skeletal tissue engineering and reconstruction. J Biomed Mater Res 43:428432

7. Garfin SR, Yuan HA, Reiley MA (2001) New technologies in spine. Kyphoplasty and vertebroplasty for the treatment of painful osteoporotic compression fractures. Spine 26(14):1511-1515

8. Heini PF, Berlemann U, Kaufmann M et al (2001) Augmentation of mechanical properties in osteoporotic vertebral bones: a biomechanical investigation of vertebroplasty efficacy with different bone cements. Eur Spine J 10:164-171

9. Kurashina K, Kurita H, Hirano M (1995) Calcium phosphate cement: in vitro and in vivo studies of the alpha-tricalcium phosphate-dicalcium phosphate dibasic-tetracalcium phosphate monoxide system. J Mater Sci Mater Med 6:340-347

10. Li C, Mason J, Yakimicki D (2004) Thermal characterization of PMMA-based bone cement curing. J Mater Sci Mater Med 15:85-89

11. Lieberman IH, Dudeney $S$, Reinhardt MK et al (2001) Initial outcome and efficacy of "kyphoplasty ${ }^{\prime \prime}$ in the treatment of painful osteoporotic vertebral compression fractures. Spine 14:1631-1638
12. Lim TH, Brebach GT, Renner SM et al (2002) Biomechanical evaluation of an injectable calcium phosphate cement for vertebroplasty. Spine 27(12):1297-1302

13. Magerl F, Aebi M, Gertzbein D et al (1994) A comprehensive classification of thoracic and lumbar injuries. Eur Spine J 3:184-201

14. Nakano M, Hirano N, Matsuura K et al (2002) Percutaneous transpedicular vertebroplasty with calcium phosphate cement in the treatment of osteoporotic vertebral compression and burst fractures. J Neurosurg 97:287-293

15. Persson T, Axén N (2003) An injectable bone void filler cement based on Ca-aluminate. Key Eng Mater 10:265-268

16. Phillips FM, Ho E, Campbell-Hupp M et al (2003) Early radiographic and clinical results of balloon kyphoplasty for the treatment of osteoporotic vertebral compression fractures. Spine 19:2260-2265

17. Uhlin T, Engqvist $H$, Jamar T (2007) Experimental study of bone integration between Xeraspine $\odot$ and sheep vertebrae. 17th Interdisciplinary Research Conference in Biomaterials, Oxford

18. Voggenreiter G, Brocker K, Röhrl B et al (2008) Ergebnisse der Ballonkyphoplastie in der Behandlung von osteoporotischen Wirbelkörperfrakturen. Unfallchirurg 111(6):403-412

19. Zarghooni K, Röllinghoff M (2009) Komplikationsanalyse bei Vertebroplastie und Kyphoplastie: Ergebnisse einer Fragebogenstudie. Deutscher Kongress für Orthopädie und Unfallchirurgie, 73. Jahrestagung der Deutschen Gesellschaft für Unfallchirurgie, 95. Tagung der Deutschen Gesellschaft für Orthopädie und Orthopädische Chirurgie, 50. Tagung des Berufsverbandes der Fachärzte für Orthopädie und Unfallchirurgie, 21.-24.10.2009, Berlin. DOI 10.3205/09dkou121 